\title{
ANÁLISIS COMPARATIVO DE LA PRECARIEDAD LABORAL EN LAS TRES PRINCIPALES METRÓPOLIS MEXICANAS PARA EL 2010*
}

\author{
SERGIO CUAUHTÉMOC GAXIOLA ROBLES LINARES* \\ EL COLEGIO DE MÉXICO
}

Recibido/ Received/ Recebido: 22/11/2012 - Aceptado/ Accepted / Aprovado: 13/04/2013

\begin{abstract}
Resumen
El objetivo general de este artículo es estudiar la precarización laboral de los trabajadores asalariados no agropecuarios en el año 2010 de las tres principales ciudades de México: Ciudad de México, Guadalajara y Monterrey. El análisis empírico de la precarización laboral se realizó mediante dos herramientas estadísticas. En primer lugar, el análisis de componentes principales que permitió reducir las dimensiones del eje analítico. En segundo lugar, el análisis por conglomerados ayudó a agrupar a la población objetivo en diferentes niveles de precarización. El documento encuentra que las ciudades analizadas exponen mercados de trabajo diferenciados con una presencia relevante de la precariedad en la estructura laboral. Se identifica así que la precariedad laboral se encuentra fuertemente arraigada en la Ciudad de México y Guadalajara, por el contrario, en Monterrey la presencia de la precariedad es menor debido a que este núcleo urbano tiene un mayor número de trabajadores en el sector industrial.
\end{abstract}

Palabras clave: Precarización laboral, Mercados de trabajo.

\section{COMPARATIVE ANALYSIS OF PRECARIOUS EMPLOYMENT IN THE THREE MAJOR MEXICANS CITIES FOR 2010}

\begin{abstract}
The objective of this article is to study the precarious employment of non - agricultural employees in 2010 of the three major Mexican cities: Ciudad de Mexico, Guadalajara and Monterrey. The empiric analysis of the precarious employment was made using two statistical tools. First, the analysis of main components that allows reducing the size of the analytic axis. Second, cluster analysis which helped to group the target population at different levels of precarization. The document presents that the cities analyzed exposed differentiated job markets with a relevant presence of the precariousness of the employment structure. Precarious employment is strongly rooted in Mexico City and Guadalajara, for the contrary, in Monterrey city the presence of precarity is lower because this urban center has a greater number of workers in the industrial sector.
\end{abstract}

Keywords: Precariousness Employment, Job Markets.

Artículo de investigación derivado del trabajo para el Grupo de Trabajo en Mercados Laborales.

Doctor en Estudios de Población por El Colegio de México. Correo electrónico: serobles99@gmail.com 


\title{
ANÁLISE COMPARATIVA DA PRECARIEDADE LABORAL NAS TRÊS PRINCIPAIS METRÓPOLES MEXICANAS EM 2010
}

\begin{abstract}
Resumo
O objetivo geral deste artigo é estudar a precarização laboral dos trabalhadores assalariados não agropecuários do ano 2010 das três principais cidades do México: Cidade do México, Guadalajara e Monterrey. A análise empírica da precarização laboral se realizou mediante duas ferramentas estatísticas. Em primeiro lugar, a análise de componentes principais, o que permitiu reduzir as dimensões do eixo analítico. Em segundo lugar, a análise por conglomerados que ajudou a agrupar a população alvo em diferentes níveis de precarização. O documento encontra que as cidades analisadas expõem mercados de trabalho diferentes com uma presença relevante da precariedade na estrutura laboral. Identifica-se assim que a precariedade laboral se encontra fortemente arraigada na Cidade do México e em Guadalajara. Em Monterrey, ao contrário, a presença da precariedade é menor devido a que este núcleo urbano tem um maior número de trabalhadores no setor industrial.
\end{abstract}

Palavras chave: Precarização laboral, Mercados de trabalho.

Gaxiola, S. (2013) Análisis comparativo de la precariedad laboral en las tres principales metrópolis mexicanas para el 2010. En: Revista de la Facultad de Ciencias Económicas de la Universidad Militar Nueva Granada. rev.fac.cienc.econ, XXI (1)

JEL: J01, J81, C38.

\section{Introducción}

A partir de la segunda guerra mundial, el trabajo constituyó un elemento de movilidad social para un segmento de los trabajadores, debido a que una parte importante de los empleos que se generaban en esa época ofrecían condiciones laborales favorables como el acceso a la atención médica, las pensiones, los contratos indefinidos, las jornadas regulares, las prestaciones laborales, etcétera. Estos beneficios establecieron las bases para que una capa de trabajadores tuviera empleos estables y seguros, otorgando certidumbre en las relaciones laborales. Según Carnoy $(2001,89)$, "en los años posteriores a la Segunda Guerra Mundial las sociedades industriales construyeron el ideal de un puesto de trabajo con jornada laboral completa en el que se trabajaba durante treinta años para una única empresa con salarios reales continuamente crecientes". ${ }^{1}$

Sin embargo, a partir de la década de los ochenta el paradigma económico a nivel mundial cambió implementándose un nuevo modelo llamado neoliberalismo ${ }^{2}$. Esto tuvo una serie de impactos en los mercados de trabajo, entre los que se destaca, un deterioro en las condiciones laborales, dando origen a fenómenos como la informalización, la precarización, la flexibilización, etcétera, los cuales han alterado las dinámicas y las relaciones laborales exponiendo a los trabajadores en una situación de mayor vulnerabilidad en la relación empleado - empleador. Es importante mencionar que estos fenómenos no son nuevos, pero se han acentuado de manera preocupante en los últimos años.

1 Es importante señalar que durante el periodo mencionado, en diversos países (principalmente de los llamados "en desarrollo") coexistieron dos grupos de trabajadores con fuertes diferencias. Por un lado, un conjunto vinculado al sector "moderno" que se caracterizaba por contar con empleos con mayor estabilidad, certidumbre y seguridad laboral. Por el otro, una masa de trabajadores marginados que se caracterizaba por empleos inestables, con bajos salarios y sin seguridad social (Pinto, 1998; Nun, 1969).

2 El modelo neoliberal está basado en una gran apertura económica y comercial, otorgando al sector privado un papel prioritario en la economía, entre otras cosas. 
En este contexto, la presente investigación optó por analizar el fenómeno de la precariedad laboral, debido a que permite desentrañar, en cierta medida, las relaciones laborales que se desarrollan al interior de los mercados de trabajo mediante el análisis de las condiciones laborales que predominan entre los trabajadores. El objetivo general de esta investigación es analizar la precarización laboral de los trabajadores asalariados no agropecuarios ${ }^{3}$ en las tres principales ciudades de México: ciudad de México, Guadalajara y Monterrey en el año 2010, con la finalidad de estudiar el nivel de precariedad de estas urbes, así como conocer las diversas modalidades que puede adquirir la precarización laboral en los mercados de trabajo urbanos en México. La elección del año 2010 se debe principalmente a dos elementos. Por un lado, resulta interesante conocer las condiciones laborales de los trabajadores asalariados no agropecuarios de las principales urbes mexicanas al finalizar la primera década del siglo XXI. Por el otro, los resultados de la encuesta que se va utilizar para este artículo (Encuesta Nacional de Ocupación y Empleo -ENOE- 2011 y 2012) son parciales, debido a que el Consejo Nacional de Población (CONAPO) en México no ha generado las proyecciones para ajustar los factores de expansión de dichas encuestas con base en el Censo 2010, lo que implica que habrá cambios en las cifras absolutas de la ENOE. ${ }^{5}$

Por último, el presente documento se estructura en cinco secciones. En primer lugar, se expone el marco teórico y metodológico de la precarización laboral. En segundo lugar, se presenta un breve contexto histórico de los mercados de trabajo de los núcleos urbanos en estudio. En tercer lugar, se muestra una configuración laboral de ciudad de México,
Guadalajara y Monterrey. En cuarto lugar se realiza el análisis de la precariedad laboral. Por último, las conclusiones de esta investigación

\section{Precariedad laboral: un debate multidimensional}

A partir de la década de los ochenta con la implementación del modelo neoliberal se generaron múltiples cambios en los mercados de trabajo (Wacquant, 2008; Gereffi, 2006; Standing, 1999). Una de las transformaciones que llamaron la atención fue el progresivo deterioro de las condiciones laborales, lo que motivó la búsqueda de nuevos conceptos que aprehendieran las modificaciones que se habían presentado en las relaciones del trabajo generadas por una diversidad de factores (Rodgers, 1989).

Según Standing (1999), un elemento que impacta en las condiciones de trabajo actuales es la "flexibilización" de las relaciones laborales en empleos que se distinguían antes por su rigidez laboral (por ejemplo sector público o algunas industrias).

En particular, se introdujo el concepto de empleo precario dentro de las discusiones sobre las condiciones de los trabajadores ${ }^{6}$. Uno de los precursores en el estudio de este concepto fue Gerry Rodgers (1989) quién vinculó el empleo precario con las formas de trabajo que caracterizaban a los trabajos inseguros e inestables.

La importancia del estudio de la precariedad laboral radica en la pérdida del trabajo estable como elemento integrador de la sociedad; pérdida que motiva la desarticulación de los derechos básicos que generaban una mayor estabilidad y seguridad social,

3 La elección de los trabajadores asalariados no agropecuarios se debe a que el fenómeno de la precariedad responde principalmente a los cambios ocurridos en las relaciones asalariadas, además, el acotar esta investigación a los trabajadores no agropecuarios se debe a un fuerte vinculo que tiene la estructura laboral de las principales metrópolis con los sectores secundarios y terciarios.

4 La elección de las tres metrópolis se debe a que las relaciones asalariadas se presentan primordialmente en las zonas urbanas, además estas tres metrópolis representan aproximadamente $30 \%$ de la población urbana, por lo que representan un panorama general de la precariedad laboral en zonas urbanas de México.

5 Para mayor información revisar la nota de resultados de la ENOE 2011 y 2012 que se encuentra en http://www.inegi.org.mx/ est/contenidos/Proyectos/encuestas/hogares/regulares/enoe/Default.aspx

6 Anteriormente predominaban las discusiones en torno a los conceptos de marginalidad e informalidad. Ya en la última década del siglo pasado, la Organización Internacional del Trabajo (OIT) acuña el concepto de "Trabajo Decente" que no ayuda mayormente para comprender a los mercados de trabajo, además dicho término se presta a juicios subjetivos. 
promoviendo una distribución más equitativa de la riqueza (Sotelo, 1999).

Un factor que ha generado inquietud en los análisis de los mercados de trabajo es el carácter expansivo que ha adquirido este fenómeno, lo que ha motivado una profunda desestabilización y segmentación del trabajo. Según Agulló (2001), la precariedad es un proceso multidimensional que transforma las relaciones laborales y se presenta en dos sentidos. Por un lado, la precariedad de la situación social que se refiere aquellos trabajadores que tienen un empleo a tiempo parcial involuntariamente, auto-empleados. Por otro, la precariedad de subsistencia, que corresponde a aquellos empleados que se encuentran por debajo de los mínimos estándares de protección social. Es importante señalar que ambas facetas no son excluyentes entre sí, sino que suelen manifestarse de manera conjunta.

De esta manera, el debate teórico de la precariedad laboral se ha enfocado en el desvanecimiento del trabajo como un elemento cohesionador al interior de la sociedad. Según Wacquant $(2000,111)$ "con el inicio de un trabajo disocializado, el empleo ya no proporciona un marco temporal y social común porque los términos del contrato son cada vez más diversos y personalizados. La permanencia en el trabajo es corto e inestable, sin protección en enfermedades y un retiro inadecuado".

En la actualidad, una de las funciones que podría estar cumpliendo la precarización laboral es mantenerse como un factor diferenciador al interior de los mercados laborales (trabajador precario versus no precario) que se refleja en el fortalecimiento de las desigualdades sociales.

Sin embargo, este concepto de empleo precario ha provocado una serie de cuestionamientos. Una de las críticas más comunes a dicho concepto menciona que las múltiples dimensiones que abarca este término generan confusión y ambigüedad en su uso, motivo por el que una serie de expertos han acotado teóricamente esta noción, limitando su población en estudio a los trabajadores asalariados.
En este contexto, diversos autores plantean el análisis de la precariedad mediante múltiples dimensiones vinculadas con las condiciones de los trabajadores, las cuales vulneran derechos básicos laborales. Por ejemplo, Rodgers (1989) define la precariedad laboral a través de elementos presentes en las relaciones laborales como la inestabilidad, la desprotección, la inseguridad y la vulnerabilidad económica y social. Para Guerra (1994), la precarización laboral debe contener al menos estas dimensiones: inseguridad, inestabilidad y bajos salarios. Otro de los autores que estudia el fenómeno de la precariedad acotada a los trabajadores asalariados es Mora (2006) que plantea este fenómeno a través de cuatro dimensiones: incertidumbre, políticas de remuneración basadas en la minimización de costos, tipo de contrato o evasión de los sistemas de seguridad social y el uso flexible de la fuerza laboral.

Un elemento que resalta en la precariedad laboral es la falta de consenso en su definición, debido a la multiplicidad de dimensiones que se asocian con las condiciones laborales. Sin embargo, el debate teórico expuesto en los párrafos anteriores evidenció que los autores coinciden en dos dimensiones: la inseguridad y la inestabilidad.

Aunado a lo anterior, los estudios de la precariedad laboral se dividen en dos vertientes. Por un lado, la conceptualización del empleo precario que analiza únicamente a los trabajadores asalariados. Por el otro, la noción de precariedad que estudia a los trabajadores asalariados y no asalariados ${ }^{7}$. Para los fines de este artículo, se optó por retomar la vertiente que analiza únicamente a los trabajadores asalariados, debido a que el concepto de precariedad responde principalmente a los cambios ocurridos en las relaciones laborales (seguridad, certidumbre, estabilidad) que se manifiesta en los trabajadores asalariados. En palabras de Mora (2006) "lo que el concepto de empleo precario intenta aprehender es el cambio en la relación social acontecido en el mundo del trabajo asalariado como resultado del inicio de una nueva fase de desarrollo capitalista".

7 Mora (2006) señala que el concepto de empleo precario que estudia a los trabajadores asalariados y no asalariados suele confundirse con las nociones de informalidad. 
Así, en esta investigación la precariedad laboral se define a través de tres dimensiones. En primer lugar, se retoma el planteamiento de Rodgers (1989) que vincula la inseguridad laboral con el acceso a la seguridad social por parte del empleo. En segundo lugar, se propone la inestabilidad laboral que Guerra (1994) asocia al tipo de contrato que rige la relación empleado-empleador. Por último, Mora (2006) plantea la política de las remuneraciones relacionadas con la minimización de costos lo que produce la contención de los salarios de los trabajadores. Esta definición expone la multidimensionalidad de la precariedad laboral y sus vínculos con elementos que difuminan al trabajo como un factor de cohesión en la sociedad, debido a que trasgrede los derechos básicos de los trabajadores.

Uno de los aspectos más complejos de la precariedad laboral es su abordaje empírico, debido a la multidimensional de dicho fenómeno. En este sentido, este trabajo plantea aproximarse al fenómeno de la precarización laboral mediante dos herramientas estadísticas. En primer lugar, se realiza el análisis por componentes principales ${ }^{8}$ que permite reducir las dimensiones del fenómeno en estudio. En segundo lugar se aplica el análisis por conglomerados que permite agrupar a los trabajadores en cuatro niveles de precariedad: precario alto, precario medio, precario bajo y no precario.

El uso de estas técnicas estadísticas en el estudio de la precariedad laboral no es nuevo, debido a que una serie de investigadores han aplicado estas herramientas por la facilidad que presentan su interpretación, además que ayuda a sintetizar de manera clara un fenómeno de naturaleza multidimensional, algunos de los autores que han utilizado estas herramientas estadísticas son Guerra (1994) y Mora (2006).

En la actualidad, la precarización se ha destacado como uno de los conceptos que ha permitido captar la complejidad de las relaciones laborales en los mercados de trabajo mundiales, debido, entre otras cosas, a su enfoque multidimensional que permite aprehender las nuevas dinámicas laborales. Retomando el planteamiento de los autores anteriores, esta investigación define la precariedad laboral mediante tres dimensiones: inseguridad (Rodgers, 1989), inestabilidad (Guerra, 1994) y bajos salarios (Mora, 2006).

\section{Metodología}

El análisis empírico de la precariedad laboral se complejiza, debido a que este fenómeno aprehende múltiples dimensiones relacionadas con las condiciones laborales (tipos de contrato, acceso a la seguridad social, prestaciones laborales, etcétera) para esta investigación, la precariedad laboral se limitó a tres dimensiones teóricas que se vinculan con algunas variables empíricas que se muestran a continuación.

- Inseguridad laboral. Esta dimensión se vincula con el acceso a la atención médica de los trabajadores (y sus familias), por lo que este elemento se asocia con otorgamiento de seguridad social por parte del empleador o empresa.

- Inestabilidad laboral. En este caso se asocia con trabajos que se caractericen por una falta de continuidad laboral, generando en el trabajador un riesgo permanente de pérdida del empleo que a su vez incide en la ruptura del trabajo como fuente integradora de la sociedad. Así, en este documento se considera el planteamiento de Guerra (1994) que propone el tipo de contrato como un eje central de la inestabilidad laboral. Además, se utiliza el tamaño de la empresa como un indicador de incertidumbre laboral, debido a que en la mayoría de los casos, las empresas con menos empleados (microempresas) se asocian a una mayor inestabilidad.

- Incertidumbre económica. En las últimas décadas se ha impuesto una política de contención de salarios que ha impactado al poder adquisitivo de los trabajadores, por lo que en esta

8 Para una descripción detallada de la realización del Análisis de Componentes Principales y el Análisis por Conglomerados (véase anexo) 
investigación considero conveniente retomar la propuesta de Mora (2006) que asocia a las adversas condiciones laborales con la incertidumbre económica que son consecuencias de políticas de las empresas de la minimización de costos.
De esta manera, la precariedad laboral se define mediante cuatro variables que se vinculan a las tres dimensiones definidas anteriormente: condición de seguridad social, tipo de contrato, tamaño de la empresa y nivel salarial. En la Tabla 1 se muestra con mayor detalle las variables que se van a utilizar y las dimensiones teóricas a las que están vinculadas?.

Tabla 1. Dimensiones y variables de la precariedad laboral ${ }^{10}$

\begin{tabular}{|c|c|c|c|c|}
\hline Dimensiones & $\begin{array}{l}\text { Inseguridad laboral } \\
\text { (atención médica) }\end{array}$ & & dad laboral & $\begin{array}{l}\text { Incertidumbre } \\
\text { económica }\end{array}$ \\
\hline \multirow{5}{*}{ Variables } & $\begin{array}{l}\text { Condición de } \\
\text { seguridad social }\end{array}$ & Tipo de contrato & $\begin{array}{c}\text { Tamaño de } \\
\text { empresa } \\
\text { o establecimiento }\end{array}$ & $\begin{array}{c}\text { Nivel } \\
\text { de ingresos }\end{array}$ \\
\hline & $\begin{array}{l}\text { 1. Con seguridad } \\
\text { social }\end{array}$ & $\begin{array}{l}\text { 1. Base, planta } \\
0 \text { indefinido }\end{array}$ & $\begin{array}{l}\text { 1. } 16 \text { y más } \\
\text { trabajadores }\end{array}$ & $\begin{array}{l}\text { 1. Más de } 5 \text { salarios } \\
\text { mínimos }\end{array}$ \\
\hline & $\begin{array}{l}\text { 2. Sin seguridad } \\
\text { social }\end{array}$ & 2. Temporal & $\begin{array}{l}\text { 2. } 6 \text { a } 15 \\
\text { trabajadores }\end{array}$ & $\begin{array}{l}\text { 2. Más de } 3 \text { hasta } \\
5 \text { salarios mínimos }\end{array}$ \\
\hline & & 3. Sin contrato & $\begin{array}{l}\text { 3. Hasta } 5 \\
\text { trabajadores }\end{array}$ & $\begin{array}{l}\text { 3. Más de } 1 \text { hasta } 3 \\
\text { salarios mínimos }\end{array}$ \\
\hline & & & 4. No especificado & $\begin{array}{l}\text { 4. Hasta } 1 \text { salario } \\
\text { mínimo }\end{array}$ \\
\hline
\end{tabular}

\section{Breve contexto histórico de los mercados de trabajo de las tres principales metrópolis de México: ciudad de México, Guadalajara y Monterrey}

Al realizar un estudio de los mercados laborales entre diferentes urbes es importante exponer un contexto histórico de cada una de las ciudades estudiadas con la finalidad de conocer su estructura, dinámica, así como las transformaciones que han ocurrido en su mercado de trabajo a lo largo del tiempo. En este sentido, la presente sección plantea un análisis a grandes rasgos de las dinámicas laborales que han predominado en la Ciudad de México, Guadalajara y Monterrey. Es importante señalar que estas tres metrópolis son los principales núcleos urbanos en México en materia poblacional, económica y política, por lo que su estudio resulta interesante, debido a que refleja a grandes rasgos las dinámicas que ocurren en el México urbano.

\subsection{Ciudad de México ${ }^{11}$}

La Ciudad de México se localiza en el centro del país, es considerada la capital de México y en el año 2010 era la ciudad más grande del país con aproximadamente 18.5 millones de habitantes, es importante señalar que esta urbe concentra la gran mayoría de las oficinas gubernamentales.

9 La fuente de información que se utilizó para este trabajo fue la Encuesta Nacional de Ocupación y Empleo (ENOE) 2010 en el segundo trimestre, la elección de este periodo se debe a que durante este tiempo los hogares presentan una mayor estabilidad económica. La ENOE cuenta con la información necesaria para medir la precarización laboral (condición de seguridad social, tipo de contrato, tamaño de empresa y nivel de ingresos).

10 Tabla de la investigacion.

11 En esta investigación cuando se refiere a la ciudad de México se considera también el área metropolitana. 
A partir de la segunda guerra mundial, la ciudad de México se constituyó como uno de los principales centros industriales y de servicios, debido a que en esa época ocurrieron transformaciones en los mercados laborales que consistieron en la concentración del trabajo en los principales núcleos urbanos, lo que generó que una parte importante de las poblaciones rurales emigrarán a las ciudades (Ward, 1991).

Sin embargo, a partir de la década de los ochenta con la implementación del modelo neoliberal ocurre una desconcentración industrial en la ciudad de México (principalmente en la manufactura), debido a que la política económica de apertura comercial del nuevo paradigma económico obligó a las empresas a una restructuración de diversas industrias a las zonas fronterizas (Montoya, 2010; Sobrino, 2000).

En este sentido, para los años noventa, en la ciudad de México se consolidaban una serie de modificaciones económicas (como consecuencia de la implementación del modelo neoliberal) que se reflejaron en la predominancia del sector terciario en el mercado laboral de esta urbe. En particular, la ciudad de México se especializó en el comercio y los servicios al productor (servicios financieros, seguros, inmobiliarios) (Garza, 2006). Así, en la actualidad la ciudad de México concentra gran parte de las actividades terciarias del país.

\subsection{Guadalajara}

Guadalajara se encuentra ubicada en el occidente de México y es la capital de la entidad federativa denominada Jalisco en el año 2010 contaba con 4.052.683 habitantes. Esta urbe es una de las tres principales metrópolis de México. Diversos autores (Alba, 2004; García \& Oliveira, 2001; González de la Rocha, 1995) señalan que durante la primera mitad del siglo XX este núcleo urbano se caracterizó por tener una estructura laboral con predominancia de pequeñas industrias. Sin embargo, a partir de 1940 con la implementación del modelo de Indus- trialización por Sustitución de Importaciones (ISI) ${ }^{12}$, la producción en esta ciudad de occidente se concentró en bienes industriales de consumo final, fortaleciendo el mercado interno (Alba, 2004).

A inicios de los años ochenta se presentan una serie de transformaciones en el mercado de trabajo, una de las primeras transformaciones laborales en Guadalajara fue la instalación de maquiladoras con la finalidad de minimizar los costos laborales mediante la mano de obra barata que se contrata en México.

En particular, la industria electrónica ha sido beneficiada con la apertura comercial, para Alba (2004) esta industria se asocia con las tecnologías de la información, teniendo un auge importante en los últimos años.

Según García \& Oliveira (2001), una característica que ha sobrevivido en el mercado laboral de Guadalajara es la fuerte presencia de pequeñas empresas que se han adaptado a las nuevas demandas de los mercados mundiales, lo anterior ha derivado en la presencia de una fuerte heterogeneidad empresarial al interior del mercado laboral de esta urbe.

\subsection{Monterrey}

Monterrey es la capital de Nuevo León se localiza al norte de México. En el año 2010 este núcleo urbano contaba con 3.6 millones de habitantes, el mercado laboral de esta ciudad norteña se ha caracterizado por tener una predominancia industrial, diversos autores han clasificado a Monterrey como uno de los principales centros industriales de México.

Después de la segunda guerra mundial, Monterrey se convirtió en uno de los centros industriales del país, debido a que consolidó a diversos grupos industriales que predominaban gran parte del proceso industrial (Solís, 2007). Con el cambio de modelo económico, en los años ochenta, Monterrey logró adaptarse a la apertura comercial que sufrió el país. Según González de la Rocha (1995) en Monterrey

12 Los principales objetivos de este modelo económico era el fortalecimiento del mercado interno, la industria nacional y el sector público. 
existió una transformación en la industria manufacturera con la finalidad de ser competitivos con la ola de nuevas importaciones. En los últimos años, la ciudad norteña continúa siendo un pilar industrial del país, sin embargo, esta actividad económica ha coexistido con la introducción de nuevas actividades principalmente en el sector terciario que es el mayor generador de empleos

En términos generales estas tres metrópolis representan aproximadamente 25 por ciento de la población total en el país (véase Tabla 2). Lo anterior expone que a que uno de cada cuatro habitantes de México radica en uno de estos núcleos urbanos.

Tabla 2. Número de habitantes en las ciudades estudiadas, México $(2010)^{13}$

\begin{tabular}{ccccc}
\hline & \multicolumn{2}{c}{$\begin{array}{c}\text { Tres principales } \\
\text { metrópolis (ciudad de } \\
\text { Indicadóxico, Guadalajara } \\
\text { y Monterrey) }\end{array}$} & Nivel nacional \\
\hline México & $\%$ & Total & $\%$ & Total \\
\hline $\begin{array}{l}\text { Población } \\
\text { total }\end{array}$ & 24,32 & 26.338 .265 & 100,00 & 108.292 .131 \\
\hline
\end{tabular}

En aspectos económicos, en el año 2009, las entidades federativas ${ }^{14}$ de estas metrópolis representaban aproximadamente 30 por ciento del Producto Interno Bruto (PIB). En particular, la ciudad de México tiene una contribución destacada con 17, 7 por ciento del PIB nacional. Lo anterior permite señalar la relevancia de estas metrópolis a nivel poblacional y económico, por lo que el estudio de sus mercados de trabajo nos muestra a grandes rasgos las dinámicas laborales que predominan en las zonas urbanas de México.

En este sentido, la riqueza de este estudio comparativo se basa en la heterogeneidad que presentan las urbes seleccionadas. Así, las tres ciudades anali- zadas muestran características del mercado laboral diferenciadas, por ejemplo, la ciudad de México en la actualidad concentra gran parte de su estructura en el sector terciario, debido a las transformaciones ocurridas en años anteriores. En lo que respecta a Guadalajara, esta gran urbe mantuvo una importante concentración de pequeñas y medianas empresas que fortalece la diversidad que mostraba la ciudad desde épocas antiguas. Por último, Monterrey se mantiene como uno de los principales centros industriales coexistiendo con el sector servicios que ha ido ganando terreno al interior de la estructura laboral.

\section{Configuración laboral de las tres principales metrópolis de México}

La configuración laboral de ciudad de México, Guadalajara y Monterrey es un elemento vital para analizar la precarización del mercado de trabajo, eje analítico de esta investigación, debido a que muestra a grandes rasgos las características de los trabajadores. En la Tabla 4, se expone que en el año 2010 la tasa de participación femenina más baja se ubicaba en la ciudad de México (en contraste con la más alta que se encontraba en Guadalajara). En lo que respecta a la tasa de participación masculina, en el año 2010 las tres ciudades contaban con una tasa mayor a 75 por ciento. Por otro lado, la urbe que en el año 2010 contaba con el menor nivel de desempleados es la capital de Jalisco que se ha caracterizado a lo largo del tiempo por contar con un número importante de pequeñas y medianas empresas.

Un elemento que expone un mercado de trabajo con mejores condiciones laborales es la tasa de trabajo asalariado, debido a que este tipo de empleos comúnmente presentan mayores niveles de seguridad y estabilidad. Sin embargo, es importante señalar que en las últimas décadas ha habido un deterioro en lo que respecta a los derechos laborales de este sector. En el año 2010, la

13 Fuente: Cálculos de la investigacion a partir de datos de la ENOE (2010).

14 Según el glosario del Instituto Nacional de Estadísticas y Geografía (INEGI), una entidad federativa es "la unidad geográfica mayor de la división político-administrativa del país; el territorio nacional se divide en 31 estados y un Distrito Federal”. La metrópoli de Guadalajara se localiza en la entidad de Jalisco, en lo que respecta a Monterrey se ubica en Nuevo León y el Distrito Federal es parte de la ciudad de México. 
tasa de trabajo asalariado con mayor porcentaje fue la de Monterrey que contaba con más del 70 por ciento (Tabla 3). En contraste, en ese mis- mo año, la ciudad de México mostraba los niveles más bajos de trabajo asalariado en los hombres y las mujeres.

Tabla 3. Indicadores Laborales de la Ciudad de México, Guadalajara y Monterrey (2010)15

\begin{tabular}{lcccccc}
\hline \multirow{2}{*}{$\begin{array}{c}\text { Indicadores } \\
\text { sociodemográficos }\end{array}$} & \multicolumn{2}{c}{ Ciudad de México } & \multicolumn{2}{c}{ Guadalajara } & \multicolumn{2}{c}{ Monterrey } \\
\cline { 2 - 7 } & $\%$ & Total & $\%$ & Total & $\%$ & Total \\
\hline Población total & 100,00 & 18.685 .091 & 100,00 & 4.052 .683 & 100,00 & 3.600 .491 \\
\hline Hombres & 48,00 & 8.968 .874 & 48,76 & 1.975 .996 & 49,83 & 1.794 .166 \\
\hline Mujeres & 52,00 & 9.716 .217 & 51,24 & 2.076 .687 & 50,17 & 1.806 .325 \\
\hline Grandes grupos de edad & & & & & & 23,43 \\
\hline $0-14$ & 24,23 & 4.377 .591 & 26,38 & 1.069 .211 & 872.230 \\
\hline $15-64$ & 69,39 & 12.965 .368 & 66,86 & 2.709 .610 & 69,17 & 2.490 .546 \\
\hline 65 y más & 7,18 & 1.342 .132 & 6,53 & 264.581 & 6,53 & 235.254 \\
\hline N.E. & 0,00 & 0 & 0,23 & 9.281 & 0,07 & 2.461 \\
\hline
\end{tabular}

La configuración laboral de las tres principales metrópolis de México expone particularidades en cada una de las urbes. Para la ciudad de México se muestra un mercado de trabajo con baja participación femenina (con respecto a Guadalajara y Monterrey). En el caso de Guadalajara se señalan los niveles más bajos de desempleo entre las tres ciudades. Por último, Monterrey mostró las tasas de trabajo asalariado más altas, lo que expone que la urbe norteña cuenta con un mercado de trabajo con mejores condiciones laborales que ciudad de México y Guadalajara.

\subsection{Distribución por grandes sectores económicos y ramas de actividad}

En esta sección se expone la distribución por grandes sectores económicos y ramas de actividad de ciudad de México, Guadalajara y Monterrey con la finalidad de conocer la estructura laboral de los trabajadores en estas urbes. En Tabla 4 se obser- van diferencias en los grandes sectores económicos de las principales metrópolis estudiadas. Por ejemplo, en el año 2010 la ciudad de México mostraba una predominancia del sector terciario $(75 \%$ del total de su población ocupada), este porcentaje se incrementa en la población femenina. Por el contrario, Monterrey expone los porcentajes más bajos de participación en el sector terciario (66\%). Entre sexos las diferencias se incrementan, destacando una fuerte participación de las mujeres en el sector terciario en las tres metrópolis (aproximadamente de $80 \%$ ).

En lo que respecta al sector secundario, Monterrey y Guadalajara exponen los mayores niveles de participación en dicho sector, en estas urbes, uno de cada tres trabajadores laboraban en el sector secundario (Tabla 5), lo anterior es congruente con lo que planteaban Alba (2004) y García y Oliveira (2001) que vinculaban estos núcleos urbanos con el sector industrial.

15 Fuente: Cálculos propios con base en la ENOE 2010. 
Tabla 4. Distribución porcentual de trabajadores por sectores económicos en las tres ciudades del estudio. ${ }^{16}$

\begin{tabular}{lcccccc}
\hline \multirow{2}{*}{ Indicadores laborales } & \multicolumn{2}{c}{ Ciudad de México } & \multicolumn{2}{c}{ Guadalajara } & \multicolumn{2}{c}{ Monterrey } \\
\cline { 2 - 7 } & Mujeres & Hombres & Mujeres & Hombres & Mujeres & Hombres \\
\hline Tasa de participación & 45,92 & 76,27 & 49,27 & 77,68 & 47,45 & 78,06 \\
\hline Población 0cupada & 92,84 & 92,37 & 93,65 & 94,98 & 91,32 & 93,61 \\
\hline Desempleados & 7,16 & 7,63 & 6,35 & 5,02 & 8,68 & 6,39 \\
\hline Población No Económicamente Activa & 54,08 & 23,73 & 50,73 & 22,32 & 52,55 & 21,94 \\
\hline Tasa de trabajo asalariado & 69,00 & 64,40 & 69,60 & 66,30 & 73,70 \\
\hline Pob 14 años y más & 7.733 .573 & 6.913 .929 & 1.593 .580 & 1.461 .541 & 1.420 .394 & 1.364 .111 \\
\hline
\end{tabular}

En las ramas de actividad, las tres ciudades cuentan con una estructura laboral con los mayores porcentajes en los servicios, el comercio y la industria manufacturera. En particular, en la ciudad de México se observa una distribución heterogénea en estas tres actividades (sin una concentración mayoritaria en alguna rama). Para Guadalajara y Monterrey, en el año 2010, la industria manufacturera se presentaba como una de las actividades con mayor concentración de trabajadores, debido a que uno de cada cuatro empleados laboraba en dicha actividad, lo anterior muestra la relevancia del sector industrial en estas urbes (Tabla 4).

En lo que respecta a las diferencias por sexo, las tres ciudades exponen una presencia similar en los servicios sociales con aproximadamente 20 por ciento de trabajadoras que laboraban en esa actividad. Uno de los elementos que resaltan en la Tabla 4 es la alta participación femenina en la industria manufacturera de Guadalajara (19,5\%), según Alba (2004) esta fuerte presencia de mujeres se debe a la instalación de maquilas que contratan mayormente al sexo femenino.

\subsection{Condiciones laborales}

En esta sección se analizan las condiciones laborales de la población objetivo de esta investigación a través de las dimensiones que se definieron de la precariedad laboral: inseguridad, inestabilidad $e$ in- certidumbre económica. Las cuales se relacionan con cuatro variables: condición de seguridad social, tipo de contrato, tamaño de la empresa y nivel de ingreso.

La condición de seguridad social se vincula con el acceso a la atención médica del trabajador y su familia, la importancia de esta variable radica en que la salud es un derecho laboral básico. Para mostrar en qué situación se encuentran los trabajadores de las tres metrópolis con respecto a esta variable en Tabla 5 se exponen el porcentaje de trabajadores asalariados no agropecuarios que tienen acceso a la seguridad social.

En el año 2010, en la ciudad de México $60 \%$ de sus trabajadores asalariados contaban con seguridad social, para Guadalajara este porcentaje aumenta a $68 \%$ y aproximadamente $75 \%$ de los trabajadores asalariados en Monterrey accedía a los servicios médicos. Lo anterior muestra que la metrópoli con condiciones laborales más adversas (en lo que respecta a la seguridad social) es la ciudad de México.

Por otro lado, en la Tabla 5, la variable que se asocia con el tipo de contrato es representada a través del porcentaje de trabajadores asalariados que en el año 2010 contaban con contrato indefinido, lo que implica que el empleado cuente con estabilidad laboral en su trabajo a lo largo del tiempo, reflejándose en mejores condiciones de vida para su familia.

16 Fuente: Cálculos propios con base en la ENOE 2010. 
Nuevamente, la ciudad de México muestra las peores condiciones laborales en lo que respecta al tipo de contrato, debido a que en el año 2010 solo la mitad de los trabajadores asalariados contaban con contrato indefinido, lo que implica que en la capital del país uno de cada dos trabajadores no tenía un contrato indefinido (Tabla 5). Para Guadalajara, se observa que $56 \%$ de los trabajadores asalariados no agrícolas en esa ciudad tenían contratos indefinidos. En el caso de Monterrey, el porcentaje de trabajadores asalariados con contratos indefinidos alcanza 60\%. Lo anterior implica que a grandes rasgos la ciudad de México se expone como la que tiene el menor porcentaje de trabajadores asalariados con contratos indefinidos. Por el contrario, Monterrey se exhibe como la ciudad con mejores condiciones laborales asociadas al tipo de contrato (véase Tabla 5).

La tercer variable que se analiza en esta sección es la referente al tamaño de la empresa o establecimiento, para este caso se analizaron a los trabajadores que laboran en empresas con más de cinco empleados, debido a que en las medianas y grandes empresas existen mejores condiciones laborales. Para las tres metrópolis se muestra que en el año 2010 más de $70 \%$ de los trabajadores asalariados no agropecuarios laboraban en empresas con más de cinco empleados (Tabla 5). Esto implica que la población en estudio se asocia a empresas medianas y grandes que a su vez exponen condiciones de mayor estabilidad y seguridad en las relaciones laborales.

Tabla 5. Distribución de trabajadores asalariados no agropecuarios por condiciones laborales en las ciudades estudiadas ${ }^{17}$

\begin{tabular}{|c|c|c|c|c|c|c|c|c|c|}
\hline \multirow{2}{*}{ Sectores económicos } & \multicolumn{3}{|c|}{ Ciudad de México } & \multirow[b]{2}{*}{ Mujer } & \multicolumn{2}{|c|}{ Guadalajara } & \multicolumn{3}{|c|}{ Monterrey } \\
\hline & Mujer & Hombre & Total & & Hombre & Total & Mujer & Hombre & Total \\
\hline Primario & 0,1 & 0,2 & 0,1 & 0,1 & 0,4 & 0,3 & 0,1 & 0,1 & 0,1 \\
\hline Secundario & 14,7 & 30,3 & 24,1 & 20,7 & 39,1 & 31,7 & 18,8 & 43,2 & 34,0 \\
\hline Terciario & 85,2 & 69,5 & 75,8 & 79,2 & 60,5 & 68,1 & 81,2 & 56,8 & 66,0 \\
\hline Total (100\%) & 2.346 .934 & 3.494 .232 & 5.841 .166 & 528.894 & 780.899 & 1.309 .793 & 463.165 & 764.781 & 1.227 .946 \\
\hline Rama de actividad económica & Mujer & Hombre & Total & Mujer & Hombre & Total & Mujer & Hombre & Total \\
\hline Agropecuaria & 0,1 & 0,2 & 0,1 & 0,1 & 0,4 & 0,3 & 0,1 & 0,1 & 0,1 \\
\hline Ind. Extractiva y eléctrica & 0,3 & 0,8 & 0,6 & 0,3 & 0,7 & 0,5 & 0,4 & 1,2 & 0,9 \\
\hline Ind. Manufacturera & 13,2 & 19,3 & 16,9 & 19,5 & 26,9 & 23,9 & 16,7 & 30,2 & 25,1 \\
\hline Construcción & 1,2 & 10,1 & 6,5 & 0,9 & 11,4 & 7,1 & 1,6 & 11,8 & 8,0 \\
\hline Comercio & 14,0 & 16,2 & 15,3 & 18,3 & 17,8 & 18,0 & 17,1 & 13,7 & 15,0 \\
\hline Restaurantes y servicios de alojamiento & 7,1 & 5,8 & 6,3 & 6,4 & 4,3 & 5,2 & 6,6 & 4,8 & 5,5 \\
\hline $\begin{array}{l}\text { Transportes, comunicaciones, correo y } \\
\text { almacenamiento }\end{array}$ & 4,3 & 12,0 & 8,9 & 2,7 & 7,3 & 5,4 & 2,9 & 6,6 & 5,2 \\
\hline $\begin{array}{l}\text { Servicios profesionales, financieros y } \\
\text { corporativos }\end{array}$ & 13,1 & 10,8 & 11,7 & 11,6 & 9,7 & 10,4 & 13,8 & 12,3 & 12,9 \\
\hline Servicios sociales & 20,5 & 6,2 & 12,0 & 18,7 & 5,6 & 10,9 & 20,8 & 6,2 & 11,7 \\
\hline Servicios diversos & 18,5 & 8,2 & 12,3 & 16,2 & 9,8 & 12,4 & 15,6 & 8,8 & 11,4 \\
\hline Gobierno y organismos internacionales & 7,2 & 10,0 & 8,9 & 5,1 & 5,9 & 5,6 & 4,1 & 4,3 & 4,2 \\
\hline Total (100\%) & 2.346 .934 & 3.494 .232 & 5.841 .166 & 528.894 & 780.899 & 1.309 .793 & 463.165 & 764.781 & 1.227 .946 \\
\hline
\end{tabular}

17 Fuente: Cálculos propios con base en la ENOE 2010.

"Los valores no especificados en todas las ciudades eran menores a uno por ciento por lo que se optó por eliminarlos. 
Por último, la variable que hace referencia a los ingresos se presenta como aquellos trabajadores que ganan más de tres salarios mínimos. El ingreso es un elemento que permite mejorar momentáneamente las condiciones de vida de los trabajadores. En este caso la diferencia entre la ciudad de México con Guadalajara y Monterrey es significativa, debido a que la capital del país presenta los niveles de trabajadores con menores ingresos. En contraste, los trabajadores analizados de la ciudad Monterrey presenta mejores ingresos. En suma, en esta sección se expuso las condiciones laborales de los trabajadores asalariados no agropecuarios de ciudad de México, Guadalajara y Monterrey, las cuales mostraron que la Ciudad de México es la metrópoli con peores condiciones laborales (en comparación con Guadalajara y Monterrey). Por el contrario, Monterrey destaco como la urbe con las mejores condiciones laborales.

\section{Análisis empírico de la precarización laboral}

Como se mencionó anteriormente, el análisis de la precarización laboral se realizó mediante dos técnicas estadísticas que permitieron, en primer lugar, sintetizar el carácter multidimensional de la precariedad laboral a través del análisis por componentes principales, para después agrupar mediante el análisis por conglomerados a los trabajadores analizados en cuatro grupos: precario alto, precario medio, precario bajo y no precario.

En este contexto, en el Tabla 6 se observan la dis- tribución porcentual de los trabajadores asalariados según su nivel de precariedad. Como se ha venido señalando Monterrey destaca como la metrópoli con mejores condiciones laborales, debido a que 50 por ciento de sus trabajadores asalariados no agrícolas no presentan ningún nivel de precariedad. En contraste, la ciudad de México y Guadalajara, únicamente $25 \%$ de los trabajadores son no precarios, es decir, uno de cada cuatro trabajadores no tienen ningún nivel de precariedad.

En lo que respecta a los trabajadores con precariedad alta, la ciudad de México y Guadalajara sobresalen con los mayores porcentajes (aproximadamente 25 por ciento). En el caso de Monterrey el porcentaje disminuye a $18 \%$. Lo anterior expone una situación preocupante para las ciudades de México y Guadalajara, debido a que en el año 2010 más de 70 por ciento de los trabajadores asalariados no agrícolas presentaban algún nivel de precariedad. Por el contrario, 50\% de los trabajadores analizados en Monterrey mostraban algún nivel de precariedad.

Es importante señalar que estas proporciones podrían representar el límite inferior de la precariedad en los mercados laborales de las tres metrópolis, ya que la población de estudio constituye un subgrupo "privilegiado" (trabajadores asalariados) dentro de la configuración laboral, por lo que se puede suponer que una cantidad importante de trabajadores no asalariados cuentan con condiciones laborales aún

Tabla 6. Distribución porcentual de los trabajadores asalariados no agropecuarios por nivel de precariedaden las ciudades estudiadas ${ }^{18}$

\begin{tabular}{lccccccccc}
\hline \multirow{2}{*}{ Condiciones laborales } & \multicolumn{3}{c}{ Ciudad de México } & \multicolumn{3}{c}{ Guadalajara } & \multicolumn{2}{c}{ Monterrey } \\
\cline { 2 - 9 } & Mujeres & Hombres & Total & Mujeres & Hombres & Total & Mujeres & Hombres & Total \\
\hline Con acceso a seguridad social & 59,59 & 63,61 & 61,92 & 67,62 & 68,85 & 68,34 & 71,61 & 78,30 & 75,74 \\
\hline Con contratos indefinidos & 51,03 & 55,43 & 53,58 & 56,57 & 57,13 & 56,89 & 61,84 & 61,96 & 61,91 \\
\hline $\begin{array}{l}\text { Laboran en empresas con más de cinco } \\
\text { empleados }\end{array}$ & 69,66 & 75,88 & 73,26 & 66,27 & 69,26 & 68,01 & 70,11 & 77,98 & 74,97 \\
\hline $\begin{array}{l}\text { Con ingresos superiores a tres salarios } \\
\text { mínimos }\end{array}$ & 25,11 & 30,96 & 28,50 & 23,27 & 39,00 & 32,41 & 35,94 & 49,72 & 44,45 \\
\hline
\end{tabular}

18 Fuente: Cálculos propios con base en la ENOE 2010. 
más precarias por la naturaleza de estos empleos que se caracterizan por ser altamente vulnerables.

\section{Conclusiones}

Los resultados que se obtuvieron en esta investigación permiten afirmar una serie de aspectos sobre las condiciones laborales de los trabajadores asalariados no agrícolas de ciudad de México, Guadalajara y Monterrey. En particular se exponen tres elementos que se consideran los de mayor relevancia.

En primer lugar, la precarización laboral es un fenómeno que impacta a la mayoría de los trabajadores en México sin importar la naturaleza del mercado de trabajo analizado. En particular, en esta investigación se analizaron tres configuraciones laborales distintas con el mismo resultado: mercados de trabajo precarios. Lo anterior permite señalar que en la actualidad los empleos que se están generando en los mercados de trabajo urbanos son precarios.

En segundo lugar, se resalta la similitud que presentan ciudad de México y Guadalajara en cuanto a las condiciones laborales adversas. Estas semejanzas son relevantes, ya que la estructura laboral de estas grandes urbes expone características diferenciadas. Por ejemplo, la capital del país muestra una concentración importante en el sector terciario. Para Guadalajara, una parte de los trabajadores laboran en la industria manufacturera. Así, la presencia de un alto porcentaje de trabajadores con algún nivel de precariedad en la ciudad de México se podría asociar a las características del empleo que se produce en el sector terciario (comúnmente de baja calidad). Por otro lado, en Guadalajara, las malas condiciones laborales podrían asociarse con un mercado de trabajo donde predominan las pequeñas empresas.

En tercer lugar, un elemento que sobresalió en esta investigación radica en el contraste que se presenta entre los niveles de precariedad de ciudad de México y Monterrey, debido a que la ciudad norteña expone menores niveles de precarización que podrían asociarse con un importante número de trabajadores que laboran en el sector industrial. Por el contrario, la ciudad de México muestra una estructura laboral dominada por el sector terciario lo que impacta en mayores niveles de precarización.

No obstante estas diferencias en cuanto a los niveles de precarización laboral, la presencia de este fenómeno en estos núcleos urbanos es preocupante y sugiere que la precariedad laboral se presenta de manera diferenciada en los mercados de trabajo urbanos en México. Para finalizar, es importante el planteamiento de políticas que fortalezcan los derechos laborales de los trabajadores, con la finalidad de que se produzcan empleos con mayor seguridad, estabilidad y certidumbre económica.

\section{Referencias}

Agulló, E. (2001). Entre la precariedad laboral y la exclusión social: los otros trabajos, los otros trabajadores. En: Agulló, E. \& Ovejero, A. (Coords.) Trabajo, individuo y sociedad: perspectivas psicosociológicas sobre el futuro del trabajo. Madrid: Pirámide.

Alba, C. (2004). De la industria tradicional a la industria electrónica. Actores locales y globales en Guadalajara, Jalisco. En: Estrada M. \& Labazeé, P. (Eds.) Producciones locales y globalización en los países emergentes: México, India y Brasil. México, D.F: CIESAS - IRD.

Carnoy, M. (2001). La transformación del trabajo en la nueva economía global. El trabajo flexible en la era de la información. Madrid: Alianza.

Dunteman, G. (1989). Principal Components Analysis. USA: SAGE.

ENOE (2011). Encuesta Nacional de Ocupación y Empleo 2011, Instituto Nacional de Estadística y Geografía (INEGI), México.

ENOE (2012). Encuesta Nacional de Ocupación y Empleo 2012, Instituto Nacional de Estadística y Geografía (INEGI), México.

García, B. \& Oliveira, O. (2001). Transformaciones recientes en los mercados de trabajo metropolitanos de México: 1990 - 1998. En: Estudios sociológicos, XIX (57): 653 - 689.

Garza, G. (2006). Estructura y dinámica del sector servicios en la Ciudad de México, 1960-2003. En: Gustavo Garza (coord.) La organización espacial del sector servicios en México. México, COLMEX: 115-170.

Gereffi, G. (2006). The new offshoring of jobs and global development. Geneva: International Institute for Labour Studies.

González de la Rocha, M. (1995). Restructuración social en dos ciudades metropolitanas: un análisis de grupos domésticos en Guadalajara y Monterrey. En: Estudios sociológicos, XIII (38): 261 - 281. México: COLMEX.

Guerra, P. (1994). El empleo precario y el empleo atípico: revisión bibliográfica y propuesta para el debate. Chile: Programa de Economía del Trabajo.

Montoya, A. (2010). Nuevas tendencias en el ordenamiento territorial del área central de la Ciudad de México y sus efectos sobre la segregación residencial socioeconómica. Tesis de maestría en urbanismo. México D.F., Universidad 
Nacional Autónoma de México

Mora, M. (2006). Ajuste estructural y empleo precario: el caso de Costa Rica. Tesis de doctorado en Ciencia Social. México D.F., El Colegio de México.

Nun, J. (1969). Superpoblación relativa, ejército industrial y masa marginal. En: Revista Mexicana de Sociología, V(2): 201.

Pinto, A. (1998). Naturaleza e implicaciones de la heterogeneidad estructural de América Latina. En: cincuenta años del pensamiento en la CEPAL, Textos seleccionados, Vol. 2. Chile: CEPAL / FCE.

Rodgers, G. (1989). Precarious work in western Europe: The state of the debate. Precarious jobs in labour market regulation: The growth of atypical employment in western Europe. Geneva, Switzerland: International Institute for Labour Studies - Free University of Brussels.

Sobrino, J. (2000). Participación económica en el siglo XX. En: Garza G. (ed.). La Ciudad de México en el fin del segundo milenio. México, El Colegio de México: 162-169.

Solís, P. (2007). Inequidad y movilidad social en Monterrey. Colegio de México.

Sotelo, A. (1999). Globalización y precariedad del trabajo en México. México: Caballito.

Standing, G. (1999). Global labour flexibility : seeking distributive justice. New York: St. Martin's Press.

Wacquant, L. (2000). Logics of urban polarization: the view from below. Renewing Class Analysis. Oxford: Blackwell Publishers - The Sociological Review.

Wacquant, L. (2008). Los condenados de la ciudad: gueto, periferias y estado. Buenos Aires, Republica Argentina: Siglo veintiuno editores.

Ward, P. (1991). México: una megaciudad. Producción y reproducción de un medio ambiente urbano. México: Alianza Editorial. 


\section{ANEXO}

\section{1) Análisis por Componentes Principales (ACP)}

Uno de los objetivos principales del ACP es la reducción del número de variables explicativas mediante la construcción de combinaciones lineales, lo que posibilita estudiar la correlación entre múltiples dimensiones, sintetizando las variables utilizadas en pocos factores. Es decir, al tener una fuerte correlación entre las variables de estudio una parte importante de su variabilidad se puede explicar con pocos componentes.

De esta manera, la implementación del ACP debe cumplir el supuesto de que las variables de estudio deben tener una alta correlación entre ellas, por lo que se aplican las pruebas Kaiser-Meyer-Olkin (KMO) y la esfericidad de Bartlett que plantean comprobar dicha correlación.

La primera prueba KMO consiste en contrastar las correlaciones parciales, las cuales deben ser explicadas por el resto de las variables, en este caso, un índice KMO cercano a uno expresa que es ACP es factible (Tabla 7). Por otro lado, la prueba de esfericidad de Bartlett plantea que la hipótesis nula de que la matriz de correlaciones es una matriz de identidad, lo que indica la falta de correlaciones significativas entre las variables, por lo que si se rechaza la hipótesis nula la aplicación del ACP es viable.

Tabla 7. Valores de la prueba $\mathrm{KMO}^{19}$

\begin{tabular}{cc}
\hline Valores KMO & Calidad del valor \\
\hline $1.00 \geq \mathrm{KMO}>.90$ & Excelente \\
\hline $.90 \geq \mathrm{KMO}>.80$ & Bueno \\
\hline $.80 \geq \mathrm{KMO}>.70$ & Aceptable \\
\hline $.70 \geq \mathrm{KMO}>.60$ & Regular \\
\hline $.60 \geq \mathrm{KMO}>.50$ & Malo \\
\hline $\mathrm{KMO}<.50$ & Inaceptable \\
\hline
\end{tabular}

Uno de los primeros pasos en la aplicación del ACP es realizar las pruebas de $\mathrm{KMO}$ y Bartlett que muestran la viabilidad del análisis. En este sentido, las pruebas que corresponden al indicador $\mathrm{KMO}$ son satisfactorios en las tres metrópolis. En la ciudad de México, la prueba KMO tiene como resultado 0,779 que se interpreta como un buen indicador para el uso de ACP. En Guadalajara, el valor del ACP correspondió a 0,778 que se interpreta como un indicador aceptable para realizar el índice de componentes principales. Por último, en Monterrey el valor KMO alcanzó 0,765 que se considera un valor aceptable para utilizar el ACP (véase Tabla 8). Así, la prueba KMO que las variables utilizadas para el ACP en las tres grandes urbes tienen una correlación importante entre ellas.

Tabla 8. Pruebas KMO y Bartlett para las ciudades estudiada $^{20}$

\begin{tabular}{lccc}
\hline \multicolumn{1}{c}{ Pruebas ACP } & $\begin{array}{c}\text { Ciudad } \\
\text { de México }\end{array}$ & Guadalajara & Monterrey \\
\hline Prueba KM0 & 0,779 & 0,778 & 0,765 \\
\hline Prueba Bartlett (valor p) & 0,000 & 0,000 & 0,000 \\
\hline
\end{tabular}

Por otro lado, la prueba de Bartlett indica que en la Ciudad de México, Guadalajara y Monterrey se rechaza la hipótesis nula (la matriz de correlaciones es una matriz identidad), debido a que el valor $\mathrm{p}=0,000<0,05$ (Tabla 8). Lo anterior indica que la matriz de correlaciones de estas variables es distinta a la matriz identidad, por lo que se puede mencionar que las variables en estudio de las tres metrópolis presentan correlaciones.

Las pruebas $\mathrm{KMO}$ y Bartlett exponen que las variables en estudio (condición de seguridad social, tipo de contrato, tamaño de empresa y nivel de ingresos) de Ciudad de México, Guadalajara y Monterrey están correlacionadas entre sí, por lo que es factible la aplicación del ACP. A continuación se analizan las comunalidades (extracción) que expresan la proporción de la varianza de cada variable explicada por los factores. Los valores más cercanos a uno muestran que la variable esta mejor explicada.

19 Fuente: Datos de la investigación.

20 Fuente: Cálculos propios con base en la ENOE 2010. 
Como se puede observar en la Tabla 9 las variables en estudio tienen una buena explicación por los componentes, por lo que se puede concluir que las variables elegidas son factibles para realizar el ACP. Por último se explica la varianza explicada que expresa en cuantos factores es posible reducir las variables en estudio.
Además, esta varianza expone el porcentaje que explica cada componente de la variabilidad total (Tabla 10).

Las pruebas mostradas en el anexo exponen la viabilidad del índice de componentes principales que se realizó para medir la precariedad laboral.

Tabla 9. Valores de las comunalidades, segun variable, para las ciudades estudiadas ${ }^{21}$

\begin{tabular}{lccc}
\hline \multirow{2}{*}{ Variables en estudio } & \multicolumn{3}{c}{ Comunalidades (extracción) } \\
\cline { 2 - 4 } & Ciudad de México & Guadalajara & Monterrey \\
\hline Seguridad social & 0,831 & 0,818 & 0,74 \\
\hline Contrato & 0,85 & 0,804 & 0,734 \\
\hline Tamaño de la empresa & 0,758 & 0,74 & 0,714 \\
\hline Nivel de Ingresos & 0,351 & 0,317 & 0,269 \\
\hline
\end{tabular}

Tabla 10. Tabla de la varianza explicada del análisis de componentes principales de las tres ciudades estudiadas ${ }^{22}$

\begin{tabular}{ccccccc}
\hline \multirow{2}{*}{ Componentes } & \multicolumn{2}{c}{ Ciudad de México } & \multicolumn{2}{c}{ Guadalajara } & \multicolumn{2}{c}{ Monterrey } \\
\cline { 2 - 7 } & Valores propios & Porcentaje varianza & Valores propios & Porcentaje varianza & Valores propios & Porcentaje varianza \\
\hline 1 & 2,79 & 69,75 & 2,679 & 66,971 & 2,457 & 61,426 \\
\hline 2 & 0,74 & 18,58 & 0,775 & 19,373 & 0,821 & 20,532 \\
\hline 3 & 0,31 & 7,76 & 0,331 & 8,285 & 0,376 & 9,393 \\
\hline 4 & 0,16 & 3,92 & 0,215 & 5,37 & 0,346 & 8,648 \\
\hline
\end{tabular}

\section{2) Análisis por conglomerados}

El Análisis por Conglomerados (AC) es una técnica estadística que agrupa los casos en función del parecido o la similitud que exista entre ellos. En particular, estos grupos se realizan con base en la cercanía o lejanía de unos con otros. El objetivo de este instrumento estadístico es agrupar las observaciones de forma que los datos sean similares al interior de los grupos (mínima varianza), y que estos sean los más heterogéneos posibles entre los diversos grupos creados (máxima varianza) (Dunteman, 1989).
La aplicación del AC puede ser mediante dos métodos. Por un lado, el método jerárquico que consiste en la agrupación de un par de casos o variables a través de una medida de distancia que los asocia para formar un conglomerados (y así sucesivamente). Por otro lado, el método no jerárquico, en el cual se agrupan las observaciones con base en un número de conglomerados (fijados con anterioridad), los conjuntos se forman a partir de unos centros iniciales que configuran las observaciones con base en la proximidad del centro inicial. En particular, en esta investigación se utilizó el método no jerárquico con la técnica $\mathrm{k}$ - medias.

\footnotetext{
Fuente: Cálculos propios con base en la ENOE 2010.

Fuente: Cálculos propios con base en la ENOE 2010.
} 